\title{
L'interjection dans un corpus d'auteurs latins
}

\author{
Joseph Denooz
}

\section{Summary}

Though they have hardly been studied so far whether by ancient or modern grammarians, interjections, which make up a highly heterogeneous category. are items of discourse the use and stylistic value of which seems to depend on several factors. While the Ancients - among them Aulus Gellius - had already noticed that a particular interjection could only be used by men and some other by women, it has so far never been established that a writer or a literary genre could have an impact on the use of interjections. The present paper examines, on the one hand, the frequency with which interjections are used in a Latin corpus consisting of 1,600.000 words and, on the other, occurrences that specifically belong to prose, poetry or to this or that writer.

Étudier l'emploi des interjections peut sembler a priori peu intéressant. On en voit une preuve dans le fait que ce type de vocables a très peu retenu l'attention des linguistes, des philologues, des grammairiens ou des stylisticiens, quelles que soient les langues ou les littératures étudiées. Tout au plus trouve-t-on quelques observations éparses et superficielles sur cette catégorie de mots qui est souvent discréditée. Ainsi, au $19^{\mathrm{e}}$ siècle, le grammairien Madvig écrit" : "les interjections ne sont que de simples exclamations que certains sentiments provoquent, et qui n'expriment aucune idée, comme ah!. C'est donc improprement qu'on les appelle mots.

Au reste, si cette catégorie a été rarement étudiée par les critiques modernes, elle n'a pas davantage retenu l'attention des grammairiens anciens. On notera néanmoins que Fr. Biville a publié à propos des vocables interjectifs des articles essentiels auxquels il est utile et même indispensable de se référer ${ }^{2}$.

Avant d'examiner l'emploi de l'interjection dans un ensemble d'œuvres latines, on consultera d'abord les auteurs latins et quelques grammairiens contemporains pour voir ce qu'ils disent des interjections. On n'insistera pas sur le fait qu'Aristote, dans la Poétique, ne cite pas l'interjection puisqu'il ne connaît que quelques parties du discours ${ }^{3}$.

\footnotetext{
${ }^{1}$ Cf. Madvig 1873.

2 Cf. Biville 1996 et Biville 2002.

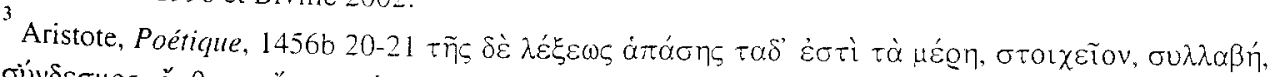

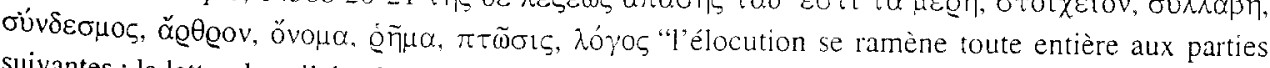
suivantes: la lettre, la syllabe, la conjonction, l'article, le nom. le verbe" (trad. Hardy, CUF, 1961).
} 
Dans les textes latins numérisés sur le CD-Rom du Packard Humanities Institute (Phi5), on dénombre 36 occurrences du mot interiectio qui se répartissent comme suit : 6 emplois chez Quintilien, une fois chez Varton et une fois la Rhétorique à Hérennius. Les autres occurrences sont dans des fragments du grammairien Quintus Remmius Palaemon (2 fois), d'Aemilius Asper ( 3 fois), de Apuleius Madaurensis : (1 occurrence), de L. Iunius Moderatus Columella ( 1 emploi) et de Maurus Servius Honoratus, le célèbre commentateur de Virgile (21 fois). Parmi les 36 emplois de interiectio, quelques-uns apportent des indications intéressantes sur la signification de ce terme. Celui-ci désigne deux faits de langue bien distincts, à savoir d'une part. les incises ou les membres de phrase placés entre parenthèses et, d'autre part, la catégorie grammaticale "interjection".

Chez Quintilien, on trouve au livre VIII pour interiectio, membre de phrase placé entre parenthèses, le texte suivant: "La parenthèse (= interiectio), dont les orateurs et les historiens se servent fréquemment pour intercaler une pensée au milieu d'une période, embarrasse ordinairement le sens, à moins qu'elle ne soit courte"".

En outre, Quintilien propose comme exemple les deux premiers vers de l'Énéide dans lesquels il considère qu'il y a une interiectio:

Arma uirumque cano. Troiae qui primus ab oris

Italiam fato profugus Laumiaque uenit.

Selon l'auteur du De Institutione oratoria, les mots fato profugus constituent une interiectio car ils créent une solution de continuité entre Italiam et Lauiniaque.

Les six occurrences d'interiectio chez Quintilien désignent trois fois une parenthèse et s'appliquent dans trois cas à la catégorie grammaticale. Parmi ceux-ci, deux sont assez explicites. Ainsi, au livre I, là où l'on trouve une énumération des catégories grammaticales, Quintilien, après avoir cité les parties du discours (substantifs, verbes, conjonctions, ... et articles), écrit: "Notre langue n'en exigeant pas, ils se trouvent confondus avec les autres parties ; mais à toutes celles que j'ai nommées, on a encore ajouté l'interjection",

Un deuxième extrait du livre I du De Institutione mérite d'être cité. À propos des solécismes pour les différentes catégories et notamment pour les adverbes, on trouve le texte suivant: "les mêmes fautes peuvent avoir lieu dans les différentes espèces de pronoms, d'interjections, et de prépositions"".

\footnotetext{
${ }^{4}$ Quint. inst. 8,2.15 Etiam interiectione. qua et oratores et historici frequenter utuntur ut medio sermone aliquen inserant sensum. impediri solet intellectus, nisi quod interponitur breue est.

${ }^{5}$ Quint. inst. 1.4.20 Noster semo atticulos non desiderat. ideoque in alias partes orationis spar. guntur: Sed accedit superioribus interiectio.

"Quint. 1.5.51 Hoc ampluss "intro" et "intus" loci aduerbia, "eo" tamen "intus" et "intro sum" soloecismi sunt. Eadem in diuersitate pronominum interiectionum praepositionum accident.
} 
Ces deux passages de l'Institution montre bien que l'interjection est une catégorie grammaticale au même titre que les adverbes, les conjonctions ou encore lés prépositions. Interiectio ne retiendra notre attention ni dans la Rhétorique à Hérennius, ni chez Varton, ni chez Columelle : aucun de ces textes n'apporte de précisions nouvelles par rapport à ce qu'a écrit Quintilien.

Des fragments de Palaemon et de Maurus Servius Honoratus, on retiendra, d'une part, que les Grecs ne considéraient pas les interjections comme une catégorie grammaticale (interiectionem Graeci [...] inter partes orationis non conputant), et, d'autre part, que les interjections traduisent des sentiments (adfectum animi) ${ }^{7}$. Ce serait d ailleurs, selon Servius, parce que cette catégorie de mots n'exprime rien d'autre que des sentiments qu'elle figure en dernier lieu dans la classification des latins : ...interiectio ideo ultima posita est, quod nihil aliud exprimit nisi affectus animi. Une remarque de Palaemon précise que le mot $o$ contribue à exprimer, lorsqu'il accompagne un pronom personnel de la première personne (ego ou nos), une affliction ou une souffrance mais pas un appel ${ }^{8}$.

Un exemple de $o$ avec un pronom personnel de la première personne exprimant l'affliction se trouve dans la tragédie Thyeste à la fin d'une longue intervention du chœur.

o nos dura sorte creatos,

seu perdidimus solem miseri, siue expulimus!

Comme on le voit, les grammairiens latins reconnaissent une catégorie grammaticale qu'ils appellent "interjection" et dont ils disent soit qu'elle traduit des sentiments, soit qu'elle contribue à l'expression du vocatif.

Chez les linguistes et les grammairiens latins modemes, on ne trouve en général que quelques remarques formelles sur les interjections ${ }^{10}$. Ainsi, la Syntaxe latine de Ernout et

\footnotetext{
${ }^{7}$ Voir par exemple Quintus Remmius Palaemon: interiectiones sunt quae nihil docibile habent: significant tamen adfectum animi, velut "heu" "eheu" "hem" "ehem" "eho" "hoe" "pop" "papae" "at" "attatae". Voir aussi Maurus Servius Honoratus, interiectio dicta est, quia interponitur ad exprimendos animi affectus, ut puta "hen mortuus est" ou encore chez le même: primo enim debemus scire, quale res coniungantur, tunc unde coniungantur: interiectio ideo ultima posita est. quod nihil alitud exprimit nisi affectus animi, qui raro interponuntur.

${ }^{8}$ Regula: prima persona, quae dicitur ego, tam in singulari quam in plurali numero non habet vocativum casum, quia nemo se vocat. sed cum forte contigerit ut dicamus o ego, o nos, non tam pronominis ratione quam interiectionis, id est dolendi gratia quan vocandi (Quintus Remmius Palaemon).

9énèque. Thyeste, 879-881: "O avec quelle dure destinée sommes-nous nés. infortunés, soit que nous ayons perdu le soleil. soit que nous l'ayons mis en fuite" (trad. L. Herrmann).

${ }^{10}$ En latin, des ouvrages de référence comme les grammaires de Leumann, Hofmann, Szantyr ou de Kühner, Stegmann ne contiennent à propos des interjections aucune remarque digne d'être retenue.
} 
Thomas ", ne fait que de rares allusions aux interjections lorsqu'elle traite de l'emploi des cas. On lit par exemple que "la particule $o$, en vieux latin et dans la prose classique, donnait un caractère affectif ou pathétique à l'expression [...]. Mais cette particule fut ensuite employée sans nuance spéciale surtout chez les poètes". À propos de l'accusatif, le même traité de syntaxe note que dans les exclamations, un mot à l'accusatif est "souvent précédé d'une particule". On peut d'ailleurs se demander si c'est à dessein que les auteurs de la Syntaxe emploient le mot "particule" pour désigner $o$, em. en....

Un autre exemple du sort réservé aux interjections se trouve dans la Grammaire de base du latin où figure un paragraphe consacré à l'interjection. On peut lire que celle-ci "est une brève exclamation, un cri qui souligne le sentiment de celui qui parle. sans s'incorporer au discours, pas plus que le vocatif. L'interjection, comme l'apostrophe. échappe à toute dépendance grammaticale et n'influence pas les autres mots de la phrase."iz

La Grammaire nouvelle de la langue latine ${ }^{13}$, ouvrage récent, consacre une page à l'interjection. On peut lire notamment que "I'interjection est une innovation des latins en tant que catégorie à part entière ... elle désigne une réaction affective du locuteur". Cette même grammaire propose quelques exemples d'interjections et note leur valeur affective. Ainsi, $\boldsymbol{o}$ aurait comme valeur affective, soit l'appel, soit l'indignation. soit encore l'admiration. Si ces remarques ont le mérite d'être plus nuancées et plus développées que ce que l'on trouve ailleurs, elles restent néanmoins très fragmentaires. On retiendra encore que les auteurs de la Grammaire nouvelle notent que certaines interjections viennent d'impératifs. D'autre part, ils classent dans cette catégorie grammaticale les jurons: hercule, Edepol, ... Ecastor!, etc. Enfin, pour se limiter à quelques ouvrages seulement, on consultera aussi la grammaire Usus de Marius Lavency ${ }^{1+}$, où l'on trouve pour l'interjection un texte très court, à savoir qu'elle: "est un mot invariable, jamais subordonné, capable de constituer à lui seul une phrase".

A partir de ces indications rares et peu significatives sur l'interjection dans la langue latine, nous allons examiner l'emploi de cette catégorie dans un corpus de quelque 1.730.000 mots répertoriés dans des cuvres appartenant aux principaux genres de la littérature latine. Au point de vue quantitatif, la catégorie des interjections est la moins employée dans la littérature latine. Sur la population étudiée. on dénombre 3.011 occurrences d'interjections. c'est-à-dire $0,17 \%$ de l'ensemble du corpus. Elles se répartissent en 729 en prose et 2.282 en poésie. Étant donné que le corpus compte 1.225 .061 occurrences

\footnotetext{
"Cf. Ernout-Thomas 1964: 15 et 22-23.

${ }^{12}$ Cf. Michel 1962:151.

${ }^{13}$ Guisard-Laizé 2001

${ }^{14}$ Lavency 1997.
} 
d'cuvres en prose et 503.407 mots provenant de textes poétiques, on voit que la prose compte $0,06 \%$ d'interjections alors que la poésie atteint $0.45 \%$. c'est-à-dire 7,5 fois plus.

Catégorie la moins fréquemment utilisée, elle est aussi une catégorie qui comporte peu de vocables différents : on en dénombre quarante-huit ${ }^{15}$ dans le corpus étudié. L'examen de ces mots et leur distribution entre auteurs conduisent à plusieurs remarques.

Tout d'abord, on observe que les quarante-huit vocables peuvent se répartir en plusieurs types. En premier lieu on retiendra les jurons et les formules de serment comme ecastor, edepol, Hercule, mehercule, mecastor, mediusfidius, pol, qui représentent 674 occurrences, soit plus de $22 \%$ du total de la catégorie et dont l'emploi. comme nous le verrons, semble être propre à certains niveaux de langue. On peut citer ensuite des mots qui sont des incitations, des encouragements, des exhortations à agir ou à réagir tels age, agedum, agite, cedo, qui sont à l'origine des formes d'impératif. Dans une troisième classe figurent des lexies qui servent à saluer, ce sont aue, chaere, qui sont utilisées pour interpeller eho, io, o, ohe. Ces dernières ont dans certains emplois une connotation affective.

Les autres interjections ont presque toutes une ou plusieurs connotations affectives. On trouve des mots qui sont des onomatopées parmi lesquelles on peut classer ha (ha! ha! pour le rire), st! pour appeler au silence. On rencontre aussi des termes comme ah, uah qui marquent tantôt la douleur, tantôt la joie ou encore l'étonnement. Plusieurs vocables ont pour but de marquer l'admiration, d'encourager; c'est le cas babae, ei, eia, eu, euge, eugepae, euax, macte, papae. On peut déduire de ce qui précède que toutes les interjections ne sont pas porteuses de sentiments, d'affectus, contrairement à ce qu'affirment tous les grammairiens anciens et la plupart des modernes.

L'observation selon laquelle la poésie contient globalement plus d'interjections que la prose conduit à poser deux questions. Est-ce vrai pour tous les poètes et pour tous les prosateurs? Certains vocables sont-ils propres au genre littéraire, sont-ils spécifiques à certains auteurs, sont-ils caractéristiques du niveau de langue?

Le tableau 1 ci-dessous présente les différentes parties du corpus selon l'ordre décroissant des fréquences des interjections.

\begin{tabular}{|l|l|l|l|}
\hline Auteur & \% interj. & Auteur & $\%$ interj. \\
\hline Vitruve & 0,000 & Ausone & 0.160 \\
\hline César (ps.) & 0.003 & Ovide & 0.266 \\
\hline Tacite & 0,018 & Properce & 0.321 \\
\hline Caton & 0,020 & Horace & 0.330 \\
\hline Salluste & 0,031 & Virgile & 0.344 \\
\hline Lucrèce & 0,046 & Tibulle & 0.421 \\
\hline Quinte-Curce & 0.050 & Sénèque (théâtre) & 0.476 \\
\hline
\end{tabular}

15 Dans l'ensemble des comédies de Plaute, qui utilise volontiers les interjections, on ne dénombre que 56 vocables (Cf. Maniet 1969). 


\begin{tabular}{|l|l|l|l|}
\hline Sénèque (prose) & 0.080 & Perse & 0.905 \\
\hline Cicéron & 0.087 & Catulle & 1.005 \\
\hline Juvénal & 0.135 & Plaute & 1.420 \\
\hline
\end{tabular}

Tableau 1: classement des auteurs selon la fréquence des interjections

De manière générale, on constate que ces mots sont moins fréquents en prose qu'en poésie. C'est dans les textes historiques ${ }^{16}$ ou techniques (Vitruve) que les interjections sont les moins nombreuses, ce qui se comprend dans la mesure où ces cuvres qui tendent à l'objectivité, évitent de traduire des sentiments, des impressions, des affectus. On remarque la proximité de Cicéron et de Sénèque. En outre. le fait que le poème philosophique de Lucrèce se situe parmi les prosateurs ne fait que confirmer ce que l'on a déjà observé à propos du De natura rerum, à savoir que ce poème didactique et philosophique présente des caractéristiques qui témoignent d’un niveau de langue intermédiaire entre prose et poésie ${ }^{17}$.

Après les prosateurs, on trouve deux groupes assez homogènes; dans le premier figurent Ovide, Properce et Horace, dans le second Virgile. Tibulle et le théâtre de Sénèque. Enfin, viennent Catulle et Plaute. Chez ce demier la fréquence des interjections n'est nullement surprenante : la langue parlée et populaire des comédies et les effets comiques appellent le recours à ce type de mots.

Pour les Camina de Catulle, il y aurait lieu d'étudier en détail l'emploi des interjections. Je me contenterai de citer ici Henri Bardon ${ }^{18}$ lorsqu'il souligne la qualité des analyses et du style de Catulle que l'on doit à Al. Ronconi : "Il a mis l'accent sur leur caractère familier [...] les moyens d'expression sont aptes à exprimer tous les sentiments, des plus raffinés aux plus brutaux".

Les variations de fréquence des interjections conduisent à examiner d'abord quels sont les vocables utilisés en prose et en poésie. Dans le tableau 2 ci-dessous, on a imprimé en caractères normaux les mots qui apparaissent en prose et en poésie, ceux qui figurent uniquement dans des cuvres poétiques sont en gras et le mot qui n'est employé qu'en prose est en italique.

Une seule lexie mediusfidius est propre à la prose. Les quarante-sept autres sont toutes utilisées par les poètes. Parmi celles-ci. trente ne sont pas employées par les prosateurs. En outre. pour les dix-sept mots communs, on remarque que le nombre d'emplois en poésie est toujours plus élevé qu'en prose, exception faite pour le juron mehercule, plus fréquent en prose et pour macte qui apparaît une fois de part et d'autre.

\footnotetext{
${ }^{16}$ César nemploie aucune interjection dans le Bellum Gallicum et le Bellum ciulle.

${ }^{17}$ En ce qui concerne Lucrèce. of. Denooz 1988 a et Denooz 1988 b.

is Cf. Bardon 1970: 12.
} 
On notera aussi que edepol ( $120 \mathrm{chez}$ Plaute, 1 fois chez Cicéron) n'apparaît en fait que chez les poètes car l'occurrence trouvée chez Cicéron se trouve dans une citation du poète Caecilius.

Dans les mots qui sont spécifiques à la poésie, aue n'est employé que chez Ovide ${ }^{19}$, chaere chez Perse, ha chez Juvénal, hei chez Tibulle, aha, ecastor, ehem, ellum, eugepae, mecastor, uah, apage, attatae, babae, eho, euax, fui, oh et st sont spécifiques aux comédies de Plaute qui contiennent la moitié des vocables qui n'apparaissent qu'en poésie.

\begin{tabular}{|c|c|c|c|c|c|c|c|c|c|}
\hline Vocable & $\begin{array}{l}\text { Eff. } \\
\text { total }\end{array}$ & $\begin{array}{l}\mathrm{P}=\text { prose } \\
\mathrm{p}=\text { poésie }\end{array}$ & $\begin{array}{l}\text { Effect. } \\
\text { prose }\end{array}$ & $\begin{array}{l}\text { Effect. } \\
\text { poésie }\end{array}$ & Vocable & $\begin{array}{l}\text { Eff. } \\
\text { total }\end{array}$ & $\begin{array}{l}\mathrm{P}=\text { prose } \\
\mathrm{p}=\text { poésie }\end{array}$ & $\begin{array}{l}\text { Effect. } \\
\text { prose }\end{array}$ & $\begin{array}{l}\text { Effect. } \\
\text { poésie }\end{array}$ \\
\hline Age & 167 & $\mathrm{Pp}$ & 39 & 128 & Evax & 4 & $\mathrm{p}$ & 0 & 4 \\
\hline Agedum & 38 & $\mathrm{Pp}$ & 15 & 23 & Evoe & 8 & $p$ & 0 & 8 \\
\hline Agite & 6 & $p$ & 0 & 6 & Fui & 2 & $\mathrm{p}$ & 0 & 2 \\
\hline $\mathrm{Ah}$ & 116 & $\mathrm{Pp}$ & 1 & 115 & $\mathrm{Ha}$ & 1 & $\mathrm{p}$ & 0 & 1 \\
\hline Aha & 4 & $p$ & 0 & 4 & Hei & 3 & $p$ & 0 & 3 \\
\hline Apage & 9 & $\mathrm{p}$ & 0 & 9 & Hem & 55 & $\mathrm{Pp}$ & 3 & 52 \\
\hline Atat & 12 & $\mathrm{Pp}$ & 1 & 11 & mehercule & 397 & $\mathrm{Pp}$ & 202 & 195 \\
\hline Attatae & 5 & $\mathrm{p}$ & 0 & 5 & Heu & 148 & $\mathrm{Pp}$ & 2 & 146 \\
\hline Ave & 1 & $\mathrm{p}$ & 0 & 1 & Heus & 33 & $\mathrm{Pp}$ & 1 & 32 \\
\hline Babae & 1 & $\mathrm{p}$ & 0 & 1 & Io & 51 & $p$ & 0 & 51 \\
\hline Cedo & 51 & $\mathrm{Pp}$ & 23 & 28 & Macte & 2 & $\mathrm{Pp}$ & 1 & 1 \\
\hline Chaere & 1 & $\mathrm{p}$ & 0 & 1 & Malum & 19 & $\mathrm{Pp}$ & 7 & 12 \\
\hline Ecastor & 38 & $\mathrm{p}$ & 0 & 38 & Mecastor & 5 & $\mathrm{p}$ & 0 & 5 \\
\hline Ecce & 267 & $\mathrm{Pp}$ & 50 & 217 & Mediusfidius & 19 & $\mathrm{P}$ & 19 & 0 \\
\hline Edepol & 121 & $\mathrm{Pp}$ & 1 & 120 & $\mathrm{O}$ & 924 & $\mathrm{Pp}$ & 315 & 609 \\
\hline Ehem & 1 & $\mathrm{p}$ & 0 & 1 & $\mathrm{Oh}$ & 10 & $\mathrm{p}$ & 0 & 10 \\
\hline Eheu & 19 & $\mathrm{Pp}$ & 1 & 18 & Ohe & 7 & $\mathrm{p}$ & 0 & 7 \\
\hline Eho & 9 & $p$ & 0 & 9 & Papae & 4 & $p$ & 0 & 4 \\
\hline $\mathrm{Ei}$ & 67 & $\mathrm{p}$ & 0 & 67 & Pol & 94 & $\mathrm{p}$ & 0 & 94 \\
\hline Eia & 16 & $\mathrm{p}$ & 0 & 16 & Pro & 70 & $\mathrm{Pp}$ & 27 & 43 \\
\hline Ellum & 2 & $\mathrm{p}$ & 0 & 2 & St & 4 & $p$ & 0 & 4 \\
\hline En & 143 & $\mathrm{Pp}$ & 21 & 122 & Vae & 28 & $\mathrm{p}$ & 0 & 28 \\
\hline Eu & 2 & $\mathrm{p}$ & 0 & 2 & Vah & 10 & $\mathrm{p}$ & 0 & 10 \\
\hline Euge & 14 & $p$ & 0 & 14 & Totaux & 3011 & & 729 & 2282 \\
\hline Eugepae & 3 & $\mathrm{p}$ & 0 & 3 & & & & & \\
\hline
\end{tabular}

Tableau 2 : Dénombrement des vocables et répartition entre prose et poésie

Sur le plan statistique, le classement des vocables selon l'ordre décroissant de leur fréquence (voir tableau 3) d'emploi indique que le mot $o$ avec 924 occurrences représente

${ }^{19}$ Ovide, Remedia, 64 
près du tiers du nombre total de la catégorie ; la proportion étant de $43 \%$ en prose et de 27 $\%$ en poésie.

Dès ce moment, on peut proposer une première conclusion. Les relevés que nous avons obtenus et les observations qu'ils permettent démontrent que même si l'interjection n'a aucune fonction syntaxique dans le discours, la manière dont elle est utilisée varie en fonction du genre littéraire ou selon l'auteur. Rare, sinon inexistante dans la prose historique, l'interjection est plus fréquente en poésie, dans la prose philosophique de Sénèque et dans l'œuvre de Cicéron, pour laquelle les relevés comprennent surtout des discours. Enfin, si on peut comprendre assez aisément son emploi dans les tragédies de Sénèque et plus encore dans les comédies de Plaute, on ne peut risquer qu'une explication prudente pour des poètes tels que Perse et Catulle.

\begin{tabular}{|l|r|l|r|l|r|l|r|}
\hline Lexie & Eff. & Lexie & Eff. & Lexie & Eff. & Lexie & Eff. \\
\hline O & 924 & Cedo & 51 & Oh & 10 & St & 4 \\
\hline Hercules & 397 & Io & 51 & Vah & 10 & Eugepae & 3 \\
\hline Ecce & 267 & Agedum & 38 & Apage & 9 & Hei & 3 \\
\hline Age & 167 & Ecastor & 38 & Eho & 9 & Ellum & 2 \\
\hline Heu & 148 & Heus & 33 & Evoe & 8 & Eu & 2 \\
\hline En & 143 & Vae & 28 & Ohe & 7 & Fui & 2 \\
\hline Edepol & 121 & Eheu & 19 & Agite & 6 & Macte & 2 \\
\hline Ah & 116 & Malum & 19 & Attatae & 5 & Ave & 1 \\
\hline Pol & 94 & Mediusfidius & 19 & Mecastor & 5 & Babae & 1 \\
\hline Pro & 70 & Eia & 16 & Aha & 4 & Chaere & 1 \\
\hline Ei & 67 & Euge & 14 & Evax & 4 & Ehem & 1 \\
\hline Hem & 55 & Atat & 12 & Papae & 4 & Ha & 1 \\
\hline
\end{tabular}

Tableau 3: classement des vocables en ordre décroissant de la fréquence d'emploi

Dès lors, même si, comme l'affirment la plupart des grammairiens, les interjections "n'expriment aucune idée" pour reprendre les termes de Madvig, si elles "n'ont aucune fonction syntaxique", leur fréquence d'emploi est néanmoins liée au genre littéraire, elles ont une portée stylistique et peut-être même sémantique tout comme les catégories grammaticales les plus porteuses de sens. Si le choix des interjections est influencé par le genre littéraire, si on y reconnaît une valeur stylistique, on y trouve aussi des particularités d'emploi qui méritent de retenir l'attention comme en atteste un passage d'Aulu-Gelle. En effet l'auteur des Noctes Atticae affirme que le mot ecastor ne serait employé que par des femmes et que jurer par Hercules serait réservé aux hommes.

In ueteribus scriptis neque mulieres Romanae per Herculem deimant neque thiri per Castorem. Sed cur illae non iurauerint Herculem, non obscurum est, nam Herculaneo sacrificio abstinent. Cur antem uir Castorem iurantes non appellauerint, non facile dictu est. Nusquam igitur scriptum inuentre est apud idoneos quidem scriptores aut "mehercle" feninam dicere aut "mecastor" uirum; "edepol" autem. quod iusiurandum per Pollucem est. ef uno ef feminae commme est (Aulu-Gelle, Noctes Atticae, 11.6.1). 
Cette affirmation est contestée par plusieurs dictionnaires qui citent deux ou trois passages des comédies de Plaute dans lesquels ecastor serait prononcé par des hommes. Ainsi, le Grand dictionnaire de la langue latine ${ }^{20}$, signale s.v. Castor que ecastor est utilisé par des hommes dans Plaute, Asinaria, V, 2, 46, et V, 2, 80 de même que dans Casina, V, 4, 13. Le dictionnaire de Forcellini ne mentionne quant à lui que deux références (Asinaria, V, 2, 80 et Casina, V, 4, 13). Dans les éditions des collections Teubner, Oxford, Les Belles Lettres et Loeb, ces trois références désignent des vers prononcés par des femmes. Il y a donc pour chacune d'elles un problème d'apparat critique.

L'attribution de Asinaria, V, 2, 46 à une femme est proposée par l'édition que J. Naudet a publiée en 1830 . Ce dernier propose une explication qui ne résout en aucun cas la question $^{21}$ puisqu'il change la répartition des répliques de manière à attribuer à des femmes les vers où figure ecastor: "Ordinem versuum, qualem vulgatae proferunt, et distinctionem personarum paululum immutavimus, suadente Bothio ratione. Namque sic diverbium melius procedit cohaeretque. Vs. 49 in vulgatis ante 46 inserunt, qui hoc loco impedimento est, et Mecastor, dignus est dant parasito, voce mecastor non patiente, utpote quae muliebris sit obstetatio".

Une recherche sur le CD-Rom Phi5 de toutes les occurrences de ecastor (mecastor) montre que, si on excepte les grammairiens, ce juron n'est employé que dans les comédies de Plaute ( 118 fois) et de Térence ( 7 fois). Si on suit les éditions de Teubner, d'Oxford et des Belles Lettres, on trouve deux occurrences de ecastor prononcées par un homme. Il s'agit, d'une part, d'une réplique de l'esclave Sosia chez Térence, Hecyra, 611 (spero ecastor) et, d'autre part, du vers 63 de Plaute, Miles gloriosus qui est prononcé par le parasite Artotrogus ${ }^{22}$. Cet emploi ne peut être pris en compte car, en réalité, le parasite rapporte des propos prononcés par une femme.

Parmi les interjections répertoriées dans le corpus du LASLA, la plus employée est $o$; l'usage qu'en font des auteurs tels que Plaute, Sénèque dans les tragédies et Virgile dans l'Énéide est assez significatif. Ainsi, on relève des emplois qui tantôt précèdent une invocation adressée aux divinités tantôt une interpellation adressée à des humains - par exemple - dans les parties dialoguées de la tragédie ou encore dans les comédies. On constate que le premier emploi est plus fréquent chez Virgile et chez Sénèque, il l'est bien moins chez Plaute. En ce qui concerne l'invocation aux dieux et déesses, elle peut accompagner soit une prière soit une marque de respect.

Dans les comédies de Plaute, $o$ accompagne aussi des phrases ou des interpellations destinées à insulter quelqu'un ou à s'en moquer. Ainsi, au vers 814 des Bacchides, Chry-

\footnotetext{
${ }^{20}$ Theill 1866.

21 Naudet 1830.

2 Ego, mecastor, pulcher est, inquit mihi, et liberalis.
} 
sale s'adresse à Nicobule en disant: O stulte, stulte, nescis nunc uenire te. On trouvera une réplique ironique au vers 759 de la même pièce lorsque Chrysale dit à Pistoclère de rester à table et de manger, ce demier répond: o imperatorem probum, signifiant par là que l'invitation semble être un ordre venant d'un chef militaire. En outre, quel que soit l'auteur ou le genre littéraire, on constate que les affectus qu accompagne la lexie o sont variés et nombreux : colère. joie, admiration. mépris, étonnement. etc.

Dans un premier temps, ces deux ou trois exemples suffisent à montrer non seulement que l'emploi des interjections est lié au genre littéraire, mais surtout qu'il serait intéressant d'étudier d'une manière systématique et exhaustive les contextes dans lesquels ces mots apparaissent. Ainsi, on doit se demander si l'affirmation selon laquelle l'interjection n'a aucune fonction syntaxique se révèle exacte. Comment faut-il, par exemple. analyser l'usage que fait Sénèque au vers 445 de Médée de ecce qui forme avec le coordonnant atque une proposition atque ecce que l'on peut traduire, comme le fait Léon Herrmann par «Et justement la voici ». De tels emplois des interjections justifieraient aussi que l'on étudie les cas où une interjection jouant le rôle de mot introducteur peut avoir un complément.

\section{Références bibliographiques}

Bardon. Henri (1970), Catulli Carmina. Latomus, Bruxelles.

Biville. Frédérique (1996). "Le statut linguistique des interjections en latin" dans : Hannah Rosén (ed.), Aspects of Latin. Papers from the Seventh International Colloquium on Latin Linguistics (Jerusalem, 19-23 April 1993), Innsbrucker Beiträge Sprachwissenschaft n 86, Innsbruk. 209220.

Biville, Frédérique (2002). "Les modalités interjectives". Lingua Latina n 7. PUPS, Paris. 275-279.

Denooz. Joseph (1988a). "Application des méthodes d'analyse factorielle à la fréquence des catégories grammaticales en latin". Les Cahiers de l'Analyse des Données. 13, 19-40.

Denooz. Joseph (1988b), "Quelques observations sur la fréquence des substantifs et des prépositions dans la littérature latine", Revue. Informatique et statistique dans les sciences humaines, 24. 151162.

Ernout, Alfred - Thomas. François (1964), Syntaxe latine. Paris.

Guisard. Philippe - Laizé. Christelle (2001). Grammaire nouvelle de la langue latine. Rosny-sousBois.

Lavency, Marius (1997). Usus. Peeters.

Madvig. J.R. (1863). Grammaire latine. Paris.

Maniet. Albert (1969). Plaute, Lexique inerse. Listes grammaticales, Relevés divers. Oims, Hildesheim.

Michel. Jacques (1962). Grammaire de base du latin. De Sikkel. Anvers.

Naudet. J. (1830). M. Accii Plani Comocdiae cum selectis rarionm notis et noris commenariis. Lemaire. vol. 1. Paris. 